\title{
Editorial
}

\section{The new vision of International Journal of Impotence Research: The Journal of Sexual Medicine}

\author{
International Journal of Impotence Research (2003) 15, 1. doi:10.1038/sj.ijir.3900976
}

This is my first editorial as the new editor of the International Journal of Impotence Research: The Journal of Sexual Medicine. I want to take this opportunity to thank the members of the society for their continuing support of our journal, the members of the publications committee for their hard work and dedication in seeking a new direction, and the past editors Gorm Wagner and Arnold Melman and editorial board for having brought the journal to its present outstanding quality of science with the highest impact factor in sexual medicine. I also want to thank those people taking this journal into the future with me, the reviewers, the new editorial board, Nature Publishing Group and especially my associate and reviews editors, Annamaria Giraldi, Dimitrios Hatzichristou Wayne Hellstrom and Ira Sharlip, Laurie McCann my editorial assistant, and my ever-present support at home, my wife Sue. We have a tremendous task before us, but we have tremendous energy and a strong international, multidisciplinary readership supporting us.

Where do we go from here? Our long-range goal is to have the IJIRjsm published monthly. The journal will include articles on sexual dysfunction in men and women, with a planned turnaround time from submission to decision for manuscripts of 30 days. Electronic submission started in November to help achieve the 30-day goal. We plan to make all manuscripts including supplements blinded and peer reviewed. For manuscripts emanating from my institution, the reviews editor will be acting editor so that I have no access to the review process. We also plan to make the journal more user friendly with dedicated reviews under the auspices of Dimitrios Hatzichristou and an up-todate calendar section with listings of meetings on sexual medicine.

While we have a large number of reviewers with diverse professional backgrounds who have agreed to help with this process, we are always looking for more qualified reviewers. To this end if you feel you can offer expertise as a reviewer, please contact me at IJIRjsm@bu.edu. When appropriate, I would like to encourage authors to cite articles from the IJIRjsm when referencing their scientific manuscripts. Future editorials will highlight past contributions from selected subjects that relate to a particularly interesting or timely article published in that particular issue of IJIRjsm.

The seeds of our ISSIR society were planted in 1978 by Adrian Zorgniotti; however, the focus was on male erectile dysfunction. For the past 25 years, members of ISSIR have been advancing the field of sexual medicine with research on male sexual function and dysfunction. More recently, however, the development of oral pharmacotherapies for the treatment of erectile dysfunction has empowered women to demand study, diagnosis and treatment of their sexual health concerns. Recent populationbased epidemiologic studies emphasize that more women have sexual dysfunction than men. This editorial highlights an interesting review written by Roy Levin on his innovative and groundbreaking research, in conjunction with the originator of the IJIR, Gorm Wagner, on the physiology of vaginal arousal. Roy's outstanding contributions to the basic science mechanisms of sexual arousal in women include: a description of the mechanisms generating vaginal transudation during sexual stimulation, methodology to record vaginal wall oxygen tension and blood flow, an enhanced understanding of vaginal physiology during orgasm and cervical physiology during sexual stimulation, the role of vaginal $\mathrm{pH}$ in various portions of the vagina and a detailed insight into the role of various neurotransmitters involved in vaginal arousal, especially VIP.

I invite you to enjoy this article, this issue and all future publications of the International Journal of Impotence Research: The Journal of Sexual Medicine. On behalf of the editorial board and the publishers, I remind you that this is your journal, so read your journal faithfully, contribute manuscripts often, cite it routinely, and contact us whenever you feel necessary.
Goldstein, I Editor 